\title{
Clinical and economic benefit of HPV-load testing in follow-up and management of women postcone biopsy for CIN2-3
}

\author{
B Almog', R Gamzu', J Bornstein', I Levin', O Fainaru', J Niv', JB Lessing' and A Bar-Am*,I \\ 'Cenvical Pathology Unit, Department of Obstetrics and Gynecology, Lis Maternity Hospital, Tel Aviv Sourasky Medical Center affiliated to the Sackler \\ Faculty of Medicine, Tel Aviv University, Tel Aviv, Israel; ${ }^{2}$ Department of Obstetrics and Gynecology, Cervical Pathology Unit, Carmel Medical Center, \\ Haifa, Israel
}

\begin{abstract}
This study aimed to evaluate the clinical and economic implications of integrating human papilloma virus (HPV) load testing into the follow-up and management protocol of women postconisation for high-grade cervical intraepithelial neoplasia (CIN2-3). We evaluated I 30 suitable women: 63 were screened biannually by Pap smears ('conventional approach') and 67 also had HPV-load testing ('HPV approach'). More stringent criteria for undergoing colposcopy or reconisation were observed by the former group compared to the latter. Both approaches were analysed for cost effectiveness. There were 33 out of 67 (49.2\%) colposcopic referrals and 24 out of 67 (35.8\%) reconisation/hysterectomies with the 'conventional approach' compared to 9 out of 63 (I4.2\%) and 7 out of 63 (1 I.1\%) with the 'HPV approach'. Cervical intraepithelial neoplasia 2-3 residual disease was detected in 7 out of 67 (I0.5\%) and 7 out of 63 (1।.1\%) women. The 'conventional approach' had more negative colposcopic biopsies and more negative reconisation/hysterectomy histologies than the 'HPV approach'. The respective cost per detection of one case of residual disease was US\$3573 and US\$3485. The 'HPV approach' required fewer colposcopic and reconisation procedures to detect one case of residual CIN2 -3 . Its higher positive predictive value than that of cytology provided a significant decrease in false positive rates and a reduction of US\$88 per detected case.

British Journal of Cancer (2003) 89, 109-112. doi:I0.1038/sj.bjc.6601032 www.bjcancer.com

(c) 2003 Cancer Research UK
\end{abstract}

Keywords: cervical intraepithelial neoplasia; colposcopy; cost - benefit analysis; cytology; histology; human papilloma virus load

The diagnosis of high-grade cervical intraepithelial neoplasia (CIN2 - 3) bears a significant risk of developing invasive carcinoma if not treated (Pinto and Crum, 2000). The recurrence rate after cone biopsy in such cases is estimated to be between 5 and 30\% (Bjerre et al, 1976; Kolstad and Klem, 1976; Larsson et al, 1983). Early diagnosis of residual or recurrent cervical disease is one of the main objectives in the follow-up of women following cone biopsy due to CIN2-3. Three diagnostic modalities, cervical cytology, colposcopy-directed biopsy and human papilloma virus status, have been previously suggested for the follow-up of such women. Currently, cervical cytology is the main screening modality being used for triaging them for further evaluation and treatment (Montz, 2000).

Cervical cytology has significant inherent potential drawbacks. The first and most significant of them is a false negative result which precludes women with undiagnosed lesions from being referred for a proper evaluation, with the consequent obvious risk of developing invasive cervical disease. In addition, cervical cytology is associated with a substantial false positive rate that may create an overload in the work schedule of the limited available colposcopic units, causing a delay in the referral of true positive cases for colposcopic evaluation and leading to over-

\footnotetext{
*Correspondence: Dr A Bar-Am, Cervical Pathology Unit, Lis Maternity Hospital, Tel Aviv Sourasky Medical Center, Tel Aviv 64239, Israel; E-mail: eshkol@tasmc.health.gov.l

Received 19 November 2002; revised 24 March 2003; accepted 31 March 2003
}

treatment, which is inevitably accompanied by anxiety, morbidity and monetary costs (Beutner et al, 1998).

Colposcopy-directed biopsy is considered as the 'gold-standard' diagnostic modality for detecting residual disease after therapeutic conisation. Most recent reports show that the majority of lowgrade cervical intraepithelial neoplasia (CIN1 lesions) will spontaneously remit (Baron and Richart, 1970; Noumoff, 1987; Hoskins et al, 1992) and that the risk of progression to CIN3 or invasive disease is minor. Accordingly, the main objective is to detect the CIN2-3 lesion, which has a significant risk to progress to invasive disease. Thus, identifying high-grade lesions in lowgrade cytology smears becomes a matter of considerable importance.

Human papilloma virus (HPV), which is the most common viral sexually transmitted disease in the United States (Hoskins et al, 1992), has since 1977 been considered an aetiologic agent for cervical cancer (Meisels et al, 1977) and is currently unequivocally linked with the development of precancerous lesions of the cervix. High-risk HPV subtypes are associated with the presence of highgrade squamous intraepithelial lesion (HSIL) or CIN2-3 (Baron and Richart, 1970). Adding HPV testing to cytology as part of the follow-up protocol of this high-risk group of patients after they had undergone therapeutic cone biopsy due to CIN2-3 may have good predictive ability. Indeed, ter Harmsel et al (1999) concluded that persistent infection with HPV 16 is associated with a higher risk of developing CIN, which is often high grade.

Previous studies on the relation between the quantitative level of HPV DNA and histologic severity of cervical lesions have yielded controversial results. Several studies using the semiquantitative 
polymerase chain reaction analysis (PCR) have shown a correlation between HSIL and a large amount of HPV-16 DNA in cervical samples and a small amount in samples of a low-grade squamous intraepithelial lesion (LSIL) (Cuzick et al, 1992, 1994; Bavin et al, 1993). In contrast, two other studies using a Hybrid Capture assay revealed smaller amounts of viral DNA in the cervical samples of women with HSIL compared with LSIL (Farthing et al, 1994; Sun et al, 1995).

We performed this prospective cohort study in order to test our hypothesis that adding assessment of the HPV load of high-risk types as a triaging tool would enable the reduction of the number of colposcopic and reconisation procedures for the detection of one case of residual CIN2 -3 . To this end, we compared the clinical and economic implications of two different approaches to the follow-up and management of women postconisation due to CIN2 - 3 lesions, namely the 'conventional (cytology) approach' vs the 'HPV (load) approach'.

\section{MATERIALS AND METHODS}

This prospective study was carried out on a cohort of 139 consecutive patients with CIN2-3 lesions who underwent cone biopsies at the Cervical Pathology Unit of the Tel Aviv Medical Center between January 1994 and December 1998. A Pap smear had been used as the screening method for the first 72 patients (Group 1, the 'conventional approach') until October 1996, when HPV load assessment was added to the screening protocol of the remaining 67 patients (Group 2, the 'HPV approach'). The mean follow-up was 53 months (range 25-77). Five of the 72 patients in Group 1 were excluded: three failed to attend all the follow-up visits and the other two were lost to follow-up. Four of the 67 patients in Group 2 were excluded: the HPV test results were incomplete in two and the other two were lost to follow-up.

The mean age was 38.8 years (range 17-54) for the Group 1 patients and 37.4 years (range 16-51) for the Group 2 patients. The following characteristics were similar between the two study groups: contraception use, education level, marital status and positive surgical margins (the latter values were 10.4 and $11.0 \%$ for Groups 1 and 2, respectively).

The follow-up protocols as well as the indications for colposcopic and reconisation procedures are summarised in Table 1. The follow-up visits took place at 6-month intervals for up to 2 years and annually thereafter. For Group 1, the indication for colposcopy was two consecutive abnormal Pap smears. The indication for reconisation was a CIN result of any degree on colposcopic biopsy. For Group 2, the indications for colposcopy were HSIL on cytology or a high viral load on an HPV test. The indication for reconisation in this group was restricted to CIN2-3 on colposcopic biopsy. Women with low-grade lesions on cytology (atypical squamous cell of undetermined significance (ASCUS) or LSIL) and a low-load HPV, which did not indicate colposcopy, were followed by serial cytologies (at intervals of 6 months). Likewise, women with low-grade lesions on colposcopy (CIN1) were referred back for follow-up by serial cytology.
We used the type 1 Hybrid Capture Test System (Digene Corporation, Silver Spring, MD, USA). Samples for the presence of a cocktail limited to the following high-risk HPV types $(16,18,31$, $33,35,45,51,52,56)$ were collected from the external cervical os and the exocervix by a special brush applicator and transferred to the lab in a container with transfer media provided in a specific kit by Digene Corp. In the type 1 Hybrid Capture assay, light is emitted during the cleavage of the tested substrate. The intensity of the emitted light is proportional to the amount of DNA present in the examined specimen. A relative light unit (RLU) measurement greater than or equal to the cutoff value indicates the presence of HPV DNA, whereas an RLU measurement less than the cutoff value indicates the absence of an HPV DNA sequence. Based on the above principles, the following scale of HPV load was used: an RLU $<0.3$ units $=$ a negative HPV DNA test, an RLU between 0.4 and 3 units $=$ a borderline test, an RLU between 4 and 9 units $=\mathrm{a}$ low HPV DNA load test and an RLU $>10=a$ high-load HPV DNA test.

A standard cone biopsy was performed by loop excision of the transformation zone (LETZ) followed by laser vaporisation of the crater base and side walls, as previously described (Bar-Am et al, 2000). The specimen sizes usually range between $1.8 \mathrm{~cm}$ in depth and $1.5 \mathrm{~cm}$ in width.

The health insurer of each woman funded the cost of treatment and follow-up. The prices for cost per case of detected residual CIN2-3 and the cost-effectiveness analysis were based on the official prices issued by the country's national healthcare providers: a visit to the doctor $=\$ 30$, each Pap smear $=\$ 18$, colposcopy and cervical biopsy $=\$ 80$, histologic analysis for colposcopy $=\$ 50 \quad$ ( $\$ 70$ for cone histology), Hybrid Capture test $=\$ 40$ ( $\$ 70$ including doctor's visit) and cone biopsy $=\$ 80$. These are the government estimates for the cost of the procedures that were issued in order to define tariffs in the public health system (in US dollars according to the rate of exchange for local currency of May 2001). The cost per detected case was calculated accordingly.

\section{Statistics}

Significant differences in proportions were assessed by Fisher's exact test. All the statistical analyses were performed using SPSS for Windows version 8.0 (SPSS Inc., Chicago, IL, USA).

\section{RESULTS}

In all, 67 women who were treated by the 'conventional approach' (Group 1) and 63 women who were treated by the 'HPV approach' (Group 2) completed the follow-up period according to the study protocol (Table 1). Table 2 presents their clinical outcome data. The two groups were equal in terms of the number of LSIL and HSIL cases in each and the overall CIN2-3 residual disease detected by reconisation/hysterectomy.

There were significantly more colposcopic referrals (49.3 vs $14.2 \%, P<0.001)$ and reconisation referrals (35.8 vs $11.1 \%$,

Table I Triaging modalities and indications for colposcopy and reconisation for the two study groups

\begin{tabular}{lll}
\hline Procedures & Conventional approach (Group I) & HPV approach (Group 2) \\
\hline Triaging modality & Pap smear every 6 months & $\begin{array}{l}\text { Pap smear and HPV load every 6 } \\
\text { months }\end{array}$ \\
Indications for colposcopy & Two consecutive abnormal Pap smears & $\begin{array}{l}\text { HSIL in Pap smear or high viral HPV } \\
\text { load }\end{array}$ \\
Indications for reconisation & Any CIN & CIN2-3 \\
\hline
\end{tabular}

HPV = human papilloma virus; $\mathrm{HSIL}=$ high-grade squamous intraepithelial lesion; CINI, $2-3=$ cervical intraepithelial neoplasia I, 2-3. 
Table 2 Comparison between the two approaches tested

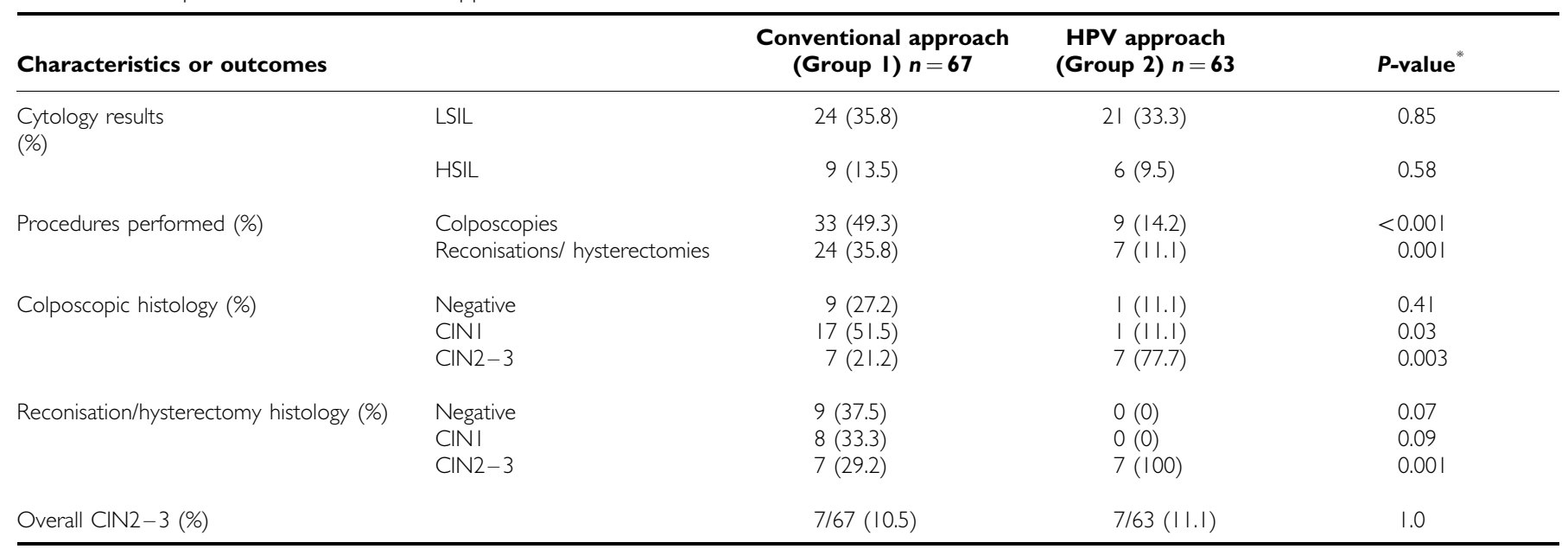

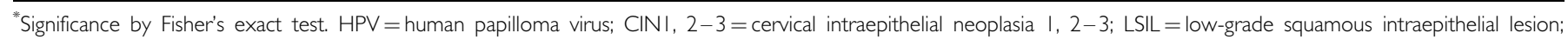
$\mathrm{HSIL}=$ high-grade squamous intraepithelial lesion.

Table 3 Positive predictive value and false positive rates of LSIL, HSIL and high-load HPV to any CIN, to $\mathrm{CINI}$ and to $\mathrm{CIN} 2-3$

\begin{tabular}{lcccc}
\hline Test & $\begin{array}{c}\text { PPV to any CIN } \\
\text { (\%) }\end{array}$ & $\begin{array}{c}\text { PPV to CIN I } \\
\text { (\%) }\end{array}$ & $\begin{array}{c}\text { PPV to CIN2-3 } \\
\text { (\%) }\end{array}$ & $\begin{array}{c}\text { False positive rate } \\
\text { (\%) }\end{array}$ \\
\hline LSIL & 33.3 & 29.1 & 4.2 & 66.6 \\
HSIL & 77.7 & 11.1 & 66.6 & 22.2 \\
HL-HPV & 100 & 0 & 100 & 0 \\
\hline
\end{tabular}

PPV = positive predictive value; CINI, 2-3=cervical intraepithelial neoplasia grade I, 2-3; HL= high-load HPV DNA; $\mathrm{LSIL}=$ low-grade squamous intraepithelial lesion; HSIL = high-grade squamous intraepithelial lesion.

$P=0.001)$ in Group 1 compared to Group 2, respectively. There was a trend of more negative colposcopic biopsies (27.2 vs $11.1 \%)$ as well as negative reconisation/hysterectomy histologies (37.5 vs $0 \%$ ) in Group 1 compared to Group 2, respectively.

Importantly, there were a total of 22 cases of LSIL or low-load HPV in Group 2 that did not indicate colposcopy, as well as one case of CIN1 on colposcopy. The total number of detected cases of CIN2 - 3 residual disease was similar between the two groups, that is, $7(10.5 \%)$ and $7(11.1 \%)$ for Groups 1 and 2, respectively.

Table 3 summarises the precision of prediction of any type of CIN by abnormal cytology or HPV load. The superiority of HPV load in the prediction of any CIN over abnormal cytology can be clearly seen. The positive predictive values (PPV) of LSIL to any CIN were relatively low $(4.2-33.3 \%)$, whereas the PPV of HSIL to any CIN residual disease or, more importantly, to CIN2 - 3 was 77.7 and $66.6 \%$, respectively. The PPV of high-load HPV for CIN2-3 was $100 \%$.

The cost of detecting one case of a CIN2 - 3 lesion according to the 'conventional approach' (Group 1) was US\$2964. If we had applied the 'conventional approach' to the whole study group, the cost-effectiveness ratio would be US $\$ 3573$. The cost of detecting one case of a CIN2 -3 lesion according to the 'HPV approach' was US $\$ 3485$. Thus, adding an HPV load assessment to the standard Pap protocol for this selected group of women would yield a decrease in cost of US $\$ 88$ per detection of one case of a residual CIN2-3 lesion.

\section{DISCUSSION}

In spite of the relatively high rate of clearance (95\% (Reich et al, 2002)), patients after conisation due to CIN2 - 3 are at a higher risk of developing residual or recurrent disease than controls. Their regular follow-up protocol after conisation includes continued cytological and colposcopic examination as well as reconisation in cases of suspected residual/recurrent lesions (Milojkovic, 2002). The optimal management of low-grade cytologies or histologies, such as ASCUS, LSIL or CIN1, in such cases remains undetermined (Livasy et al, 1999; Nagai et al, 2000; Reich et al, 2001).

The main objective of the present prospective cohort study was to analyse the clinical and economic contribution of adding an HPV-load test to the standard cytological follow-up following therapeutic conisation due to CIN2-3. Indications for advanced diagnostic procedures were allowed to be restricted whenever the HPV load was assessed (the 'HPV approach'), but they were to be expanded whenever only cytology had been used for triaging subjects (the 'conventional approach').

A comparison between the two study groups shows that HPVload evaluation identifies the presence of CIN2-3 at a detection rate equal to the standard cytological approach, but with significantly fewer colposcopies and reconisation procedures. The main contribution of an HPV-load evaluation is its ability to identify normal or low-grade lesions that might progress to highgrade lesions. Three such patients from Group 2 with normal cytologic results were further evaluated only due to the discovery of a high HPV load and they were eventually proved to be CIN2-3. Accordingly, the PPV of a high-load HPV test was maximal (i.e., $100 \%)$ according to these findings.

When an HPV-load evaluation is unavailable for the follow-up of such a high-risk group and initial triaging is performed by repeated cytologies, however, one must seriously consider further and complete histological evaluation of all ASCUS, LSIL and CIN1 results. The case of LSIL that was additionally evaluated by colposcopy and subsequently reclassified as CIN2-3 in Group 1 supports this contention. Such a policy, however, results in a high rate of colposcopic and reconisation referrals and, consequently, a 
high number of low-grade colposcopic and reconisation histologic results.

Previous studies on the relation between the HPV load and the histologic grade of cervical lesions have yielded controversial results. Several investigations in which a semiquantitative PCR was used showed a significant correlation between cytological grade and the amount of HPV-16 DNA in cervical samples (Cuzick et al, 1992, 1994; Bavin et al, 1993). In contrast, two other studies using Hybrid Capture assays revealed smaller amounts of viral DNA in cervical samples of women with HSIL compared to those with LSIL (Farthing et al, 1994; Sun et al, 1995). Sun et al (2001) recently revealed that the effect of HPV infection on SIL development is dominated by the viral load, thus highlighting a potential application of viral load testing in predicting the advancement of cervical intraepithelial lesions. Ylitalo et al (2000) reported that CIN3 is associated with HPV-16-positive women who have consistently high viral loads, and Sherman et al (2002) concluded that for women with ASCUS, HPV-load testing is highly sensitive for detecting CIN3 and cancer with dramatically fewer referrals for colposcopy.

\section{REFERENCES}

Bar-Am A, Daniel Y, Ron IG, Niv D, Kuprminc MJ, Bornstein J, Lessing JB (2000) Combined colposcopy, loop conization, and laser vaporization reduces recurrent abnormal cytology and residual disease in cervical dysplasia. Gynecol Oncol 78: 47-51

Baron BA, Richart RM (1970) A statistical model of the natural history of cervical carcinoma. II. Estimates of the transition time from dysplasia to carcinoma in situ. J Natl Cancer Inst 45: 1025-1034

Bavin PJ, Giles JA, Deery A, Crow J, Griffiths PD, Emery VC, Walker PG (1993) Use of semiquantitative PCR for human papillomavirus DNA type 16 to identify women with high grade cervical disease in a population presenting with a mildly dyskaryotic smear report. $\mathrm{Br} \mathrm{J}$ Cancer 67: $602-605$

Beutner KR, Reitano MV, Richwald GA, Wiley DJ (1998) External genital warts: report of the American Medical Association Consensus Conference. Clin Infect Dis 27: 796-806

Bjerre B, Eliasson G, Linell F, Soderberg H, Sjoberg NO (1976) Conization as only treatment of carcinoma in situ of the uterine cervix. Am J Obstet Gynecol 125: $143-152$

Cuzick J, Terry G, Ho L, Hollingworth T, Anderson M (1992) Human papillomavirus type 16 in cervical smears as predictor of high-grade cervical intraepithelial neoplasia. Lancet 339: 959-960

Cuzick J, Terry G, Ho L, Hollingworth T, Anderson M (1994) Type-specific human papillomavirus DNA in abnormal smears as a predictor of highgrade cervical intraepithelial neoplasia. $B r$ J Cancer 69: 167-171

Farthing A, Masterson P, Mason WP, Vousden KH (1994) Human papillomavirus DNA in abnormal smears as a predictor of high-grade cervical intraepithelial neoplasia. Br J Cancer 69: 67-71

Goldie SJ, Kuhn L, Denny L, Pollack A, Wright TC (2001) Policy analysis of cervical cancer screening strategies in low-resource settings: clinical benefits and cost-effectiveness. J Am Med Assoc 285: $3107-3115$

Hoskins WJ, Perez CA, Young RC (ed) (1992) Principles and Practice of Gynecologic Oncology. Lippincott: Philadelphia

Kim JJ, Wright TC, Goldie SJ (2002) Cost-effectiveness of alternative triage strategies for atypical squamous cells of undetermined significance. J Am Med Assoc 287: 2382-2390

Kolstad P, Klem V (1976) Long-term follow-up of 1121 cases of carcinoma in situ. Obstet Gynecol 48: $125-129$

Larsson G, Gullberg B, Grundsell H (1983) Comparison of complications of laser and cold knife conization. Obstet Gynecol 62: 213-217

Livasy CA, Maygarden SJ, Rajaratnam CT, Novotny DB (1999) Predictors of recurrent dysplasia after a cervical loop electrocautery excision procedure for CIN-3: a study of margin, endocervical gland, and quadrant involvement. Mod Pathol 12: 233-238
We have shown a reduced cost per detection of one case of residual CIN2-3 lesion if HPV-load assessment were to be included in addition to the standard Pap smear. Several authors have independently reported that inclusion of HPV assessment in screening for cervical cancer may be more clinically effective than Pap tests alone at higher but still 'reasonable costs' (Goldie et al, 2001; Kim et al, 2002; Mandelblatt et al, 2002). These costs were calculated for quality-of-life measures or years of life saved. Although we did not perform a complete cost-utility analysis, we believe that the distinct significance of the present results applies to the evaluation of different triaging protocols for women at high risk for CIN2-3, rather than as a primary screening protocol. To conclude, we regard the 'HPV approach' as the more reasonable choice for such a selective and high-risk group, even had our results shown a small incremental cost.

\section{ACKNOWLEDGEMENTS}

Esther Eshkol is thanked for editorial assistance.
Mandelblatt JS, Lawrence WF, Womack SM, Jacobson D, Yi B, Hwang YT, Gold K, Barter J, Shah K (2002) Benefits and costs of using HPV testing to screen for cervical cancer. J Am Med Assoc 287: 2372-2381

Meisels A, Fortin R, Roy M (1977) Condylomatous lesions of the cervix. II. Colposcopic and histopatholog. Acta Cytol 21: 379-390

Milojkovic M (2002) Residual and recurrent lesions after conization for cervical intraepithelial neoplasia grade 3. Int J Gynaecol Obstet 76: $49-53$

Montz FJ (2000) Management of high-grade cervical intraepithelial neoplasia and low-grade squamous intraepithelial lesion and potential complications. Clin Obstet Gynecol 43: 394-409

Nagai Y, Maehama T, Asato T, Kanazawa K (2000) Persistence of human papillomavirus infection after therapeutic conization for CIN 3: is it an alarm for disease recurrence? Gynecol Oncol 79: 294-299

Noumoff JS (1987) Atypia in cervical cytology as a risk factor for intraepithelial neoplasia. Am J Obstet Gynecol 156: 628-631

Pinto AV, Crum CP (2000) Natural history of cervical neoplasia: defining progression and its consequences. Clin Obstet Gynecol 43: 352-362

Reich O, Lahousen M, Pickel H, Tamussino K, Winter R (2002) Cervical intraepithelial neoplasia III: long-term follow-up after cold-knife conization with involved margins. Obstet Gynecol 99: $193-196$

Reich O, Pickel H, Lahousen M, Tamussino K, Winter R (2001) Cervical intraepithelial neoplasia III: long-term outcome after cold-knife conization with clear margins. Obstet Gynecol 97: 428-430

Sherman ME, Schiffman M, Cox JT (2002) Effects of age and human papilloma viral load on colposcopy triage: data from the randomized Atypical Squamous Cells of Undetermined Significance/Low-Grade Squamous Intraepithelial Lesion Triage Study (ALTS). J Natl Cancer Inst 94: $102-107$

Sun CA, Lai HC, Chang CC, Neih S, Yu CP, Chu TY (2001) The significance of human papillomavirus viral load in prediction of histologic severity and size of squamous intraepithelial lesions of uterine cervix. Gynecol Oncol 83: $95-99$

Sun XW, Ferenczy A, Johnson D, Koulos JP, Lungu O, Richart RM, Wright Jr TC (1995) Evaluation of the hybrid capture human papillomavirus deoxyribonucleic acid detection test. Am J Obstet Gynecol 173: $432-437$

ter Harmsel B, Smedts F, Kuijpers J, van Muyden R, Oosterhuis W, Quint W (1999) Relationship between human papillomavirus type 16 in the cervix and intraepithelial neoplasia. Obstet Gynecol 93: 46-50

Ylitalo N, Sorensen P, Josefsson AM, Magnusson PK, Andersen PK, Ponten J, Adami HO, Gyllensten UB, Melbye M (2000) Consistent high viral load of human papillomavirus 16 and risk of cervical carcinoma in situ: a nested case-control study. Lancet 355: 2179-2180 